\title{
Engajamento entre Estudantes do Ensino Superior nas Ciências da Saúde (Validação do Questionário Ultrecht Work Engagement Scale (UWES-S) com Estudantes do Ensino Superior nas Ciências da Saúde)
}

Engagement among Health Science Undergraduate Students (Validation of the Ultrecht Work Engagement
Scale (UWES-S) with Health Science
Undergraduate Students)

\author{
Juliana Ollé Mendes da Silva ${ }^{I}$ \\ Gerson Alves Pereira Junior ${ }^{I I}$ \\ Izabel Cristina Meister Martins Coelho ${ }^{I}$ \\ Gledson Luiz Picharski ${ }^{I}$ \\ Ivete Palmira Sanson Zagonel
}

\section{PALAVRAS-CHAVE \\ - Estudos de Validação. \\ - Estudantes de Ciências da Saúde. \\ - Ensino Superior. \\ - Saúde.}

${ }^{I}$ Faculdade Pequeno Príncipe, Curitiba, Paraná, Brasil.

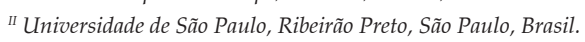

\section{RESUMO}

O ingresso na vida acadêmica traz mudanças significativas, ligadas ao modelo de ensino-aprendizagem e à rotina do estudante. O engajamento é considerado o oposto do burnout: ao contrário dos estudantes que sofrem de estresse acadêmico, os alunos engajados se sentem muito conectados às atividades, percebendo-as como um desafio positivo. Esta pesquisa teve como objetivo geral avaliar o escore de engajamento entre estudantes do ensino superior das Ciências da Saúde; e como objetivos especificos, validar o instrumento Utrecht Work Engagement Scale (UWES-S) com estudantes do ensino superior nas Ciências da Saúde; avaliar Vigor, Dedicação e Absorção do constructo engajamento e comparar os escores entre variáveis de duas ou mais categorias para obter o escore de engajamento entre estudantes do ensino superior nas Ciências da Saúde. Método: Estudo observacional analítico transversal, com abordagem quantitativa. Resultados: O UWES-S alcançou validade confirmatória ao ser aplicado a estudantes de cinco cursos da área da saúde nos níveis de Vigor, Dedicação e Absorção. No que tange ao escore das dimensões, tem-se que Medicina e Enfermagem apresentam maior Vigor e Dedicação; os turnos de estudo manhã e integral apresentam maior Vigor e Absorção; na dimensão Absorção, observou-se maior escore entre os estudantes que cursavam até o quarto período; quanto ao tempo de dedicação aos estudos fora da faculdade, observou-se que os escores se mantiveram altos nas três dimensões do constructo, assim como para estudantes que praticavam atividades de lazer. Estudantes de Medicina e Enfermagem dos turnos da manhã e integral, casados, com filhos, que dedicam grande parte do tempo aos estudos fora da faculdade, que têm atividades de lazer apresentam maior escore de engajamento com relação às demandas acadêmicas. Conclusão: O engajamento como constructo é recente no Brasil. Seu conhecimento possibilitou um novo olhar sobre o contexto acadêmico, os mecanismos de adaptação do estudante ao ensino superior e a necessidade da proximidade do docente como mediador e da universidade como sustentáculo do processo. 


\section{KEYWORDS}

- Validation Studies.

- Health Science Students.

- Undergraduate.

- Health.

\section{ABSTRACT}

Entry into academic life brings about significant changes in one's life, and these changes are linked to the teaching-learning model and the student's routine. Engagement is considered the opposite of Burnout, and unlike students who suffer from academic stress, engaged students feel very connected to the activities, perceiving them as positive challenges. This research had the general objective of evaluating the Engagement Score among Health Science undergraduate students; and, the specific objectives of validating the Utrecht Work Engagement Scale (UWES-S) instrument with Health Science undergraduate students; to evaluate vigor, dedication and absorption of the Engagement construct, as well as to compare scores between variables of two or more categories to obtain the Engagement Score among Health Science undergraduate students. Method: Cross-sectional analytical observational study with a quantitative approach. Results: The UWES-S was validated when applied to undergraduate students of five Health Science programs, in levels of vigor, dedication and absorption. Regarding the Dimensions Score, medicine and nursing students presented greater vigor and dedication; part-time morning class and full-time students show greater vigor and absorption; in the Absorption dimension, a higher score was observed among students up to the fourth semester of the course. As for the time dedicated to study outside university, the observed scores remained high in all three dimensions of the construct, as well as for students who practiced leisure activities. Part-time morning and full-time medical and nursing students who were married and have children, who spend much of their time studying outside university and maintain leisure activities, show a higher Engagement Score in relation to academic demands. Conclusion: Engagement is a recent construct to be applied to Brazil. Furthering our understanding of this construct enabled us to gain a new perspective of the academic setting, the students' mechanisms to adapt to higher education and the need for the teacher to be within reach as a mediator and the university to act in support of this process.

Recebido em: 22/11/17

Aceito em: 4/1/18

\section{INTRODUÇÃO}

$\mathrm{O}$ ingresso do estudante na vida acadêmica traz consigo mudanças significativas, que estão ligadas ao modelo de ensino-aprendizagem, bem como à rotina com que o aluno se depara ${ }^{1}$. Ajustar-se à rotina universitária implica a integração, no âmbito social, com as pessoas desse novo contexto. O desdobramento da capacidade de lidar com as emoções, a autonomia, a identidade, relações caracterizadas por maior interdependência, constituem tarefas centrais para o estudante. Entende-se, assim, que a adaptação passa a ser um processo contínuo de desenvolvimento e crescimento pessoal, acadêmico e, inclusive, profissional, para o estudante universitário, preparando-o, de certa forma, para novos contextos e experiências ${ }^{2-5}$.

O engajamento pode ser percebido no indivíduo pela energia despendida, pelo envolvimento e pela eficácia ao desempenhar determinada atividade, uma vez que seus valores são considerados seja no ambiente de trabalho ou nos estudos. As pessoas engajadas mostram-se abertas a novas experiências, sendo capazes de explorar com avidez o ambiente/situação em que se encontram, tornando-se inclusive mais criativas $s^{6,7,8}$.
Os fundamentos da psicologia positiva contribuíram para o constructo Engagement ou Work Engagement, o qual tem por objetivo fornecer uma nova abordagem acerca das potencialidades e virtudes humanas, pesquisando as condições e processos que contribuem para o progresso de indivíduos e comunidades $9,10,11$.

Quando utilizado com estudantes de ensino superior, o conceito de engajamento apresenta as mesmas características. Os estudantes engajados se sentem conectados às suas atividades e as percebem como um desafio positivo, pois variáveis como apoio social, autoeficácia, grau de satisfação pessoal, incentivo à autonomia auxiliam no desempenho e alcance dos objetivos acadêmicos ${ }^{12,13}$.

O engajamento é motivacional e, portanto, positivo, de natureza social, composto por fatores de conduta. Tais fatores constituem o instrumento desenvolvido por Schaufeli e Bakker, que teve por objetivo avaliar o engajamento na prática laboral do indivíduo ${ }^{9,8}$ e inclui Vigor, que traduz energia; Dedicação, que se refere ao emocional, e Absorção, que se refere ao aspecto cognitivo, dando origem à escala $U W E S^{14}$. 
Ao longo das últimas duas décadas, investigações acerca do engajamento entre estudantes têm sido desenvolvidas em decorrência de problemas educacionais persistentes, como baixa realização com os estudos, altas taxas de evasão, tédio estudantil e alienação ${ }^{15}$. A escala UWES-S, aplicada a estudantes, foi formulada originalmente em 2003 pelos psicólogos Schaufeli e Bakker, pesquisadores da Utrecht University (Holanda). Posteriormente, foi adaptada em vários países, como Inglaterra, Alemanha, França, Noruega, Suécia, Finlândia, Espanha, Grécia, Rússia, Portugal e Brasil. É composta por 17 questões ${ }^{7}$.

O engajamento constitui um "fator-chave" para a aprendizagem e o sucesso escolar/acadêmico, com o alcance de notas altas e maiores taxas de conclusão escolar. Por se tratar de uma "metaconstrução", inclui comportamento observável, cognição e emoções dos estudantes ${ }^{16}$.

Um estudo desenvolvido com 109 estudantes de Odontologia da Universidade de San Sebastian (Chile) articulou o escore geral de engajamento com o resultado do Questionário de Satisfação Acadêmica ${ }^{17}$ e com variáveis sociodemográficas, com o objetivo de relacionar tais escores com variáveis como sexo e ano de ingresso. Concluiu que os estudantes com maior nível de engajamento eram do sexo feminino e aqueles com mais tempo de curso. Apontou também a importância de buscar estratégias para promover o engajamento em estudantes de saúde como fator motivacional no processo de formação do futuro profissional de saúde ${ }^{18}$.

Ao se cruzarem os resultados obtidos com a escala UWES-S e o Questionário de Saúde Geral (QSG-12) ${ }^{19}$, com 1.840 estudantes de Enfermagem e Fisioterapia das universidades de Castilla e Leon (Espanha) para avaliar o nível de mal-estar psicológico e a relação do engajamento acadêmico com as variáveis sexo e formação profissional, foram observados altos níveis de Absorção e Dedicação, assim como baixos níveis de estresse psicológico, resultando em altos níveis de Vigor e Resiliência dos estudantes durante o processo de aprendizagem ${ }^{20}$.

Uma pesquisa com 202 estudantes de Psicologia da Universidade de Barranquilla (Colômbia), que analisou a associação entre percepção de estresse, engajamento e rendimento acadêmico, utilizando o inventário de burnout acadêmico e a escala UWES-S, concluiu que estudantes com níveis significativos de engajamento apresentam escores menores de estresse acadêmico ${ }^{1}$.

Com base nesta reflexão, este estudo justifica-se pela necessidade de aprofundamento no tema, considerando que a discussão a respeito deste constructo ainda se mostra incipiente e recente no Brasil, não sendo encontradas publicações que estabeleçam o nível de engajamento entre estudantes do ensino superior nas Ciências da Saúde. O uso deste constructo permite identificar os estudantes engajados com as atividades acadêmicas, com emoções positivas e, consequentemente, com a saúde física e psicológica; desvelar a interação positiva dos estudantes com a instituição de ensino; estabelecer o índice de engajamento ao longo da formação; conhecer os indicadores de satisfação, que podem supor um fator protetor diante do fracasso e abandono dos estudos. A avaliação contínua do engajamento subsidia as instituições de ensino para criar estratégias de promoção do engajamento, com base no conhecimento das variáveis intervenientes e superação do estresse acadêmico.

O objetivo geral deste estudo, resultado de pesquisa efetivada no mestrado ${ }^{21}$, foi avaliar o escore de engajamento entre estudantes do ensino superior das Ciências da Saúde e, como específicos, validar o instrumento Utrecht Work Engagement Scale (UWES-S) com estudantes do ensino superior nas Ciências da Saúde; avaliar Vigor, Dedicação e Absorção do constructo engajamento pela aplicação do UWES-S; e comparar os escores entre as variáveis estabelecidas no estudo, para obter o escore geral de engajamento entre estudantes do ensino superior nas Ciências da Saúde. A expectativa deste estudo foi de que o engajamento diminui ao longo da formação desses estudantes.

\section{PROCEDIMENTOS METODOLÓGICOS}

Estudo observacional analítico transversal, com abordagem quantitativa. As análises estatísticas foram efetivadas utilizando-se o programa estatístico R. A amostra foi constituída por 537 estudantes de cursos da área de Saúde (Enfermagem, Medicina, Biomedicina, Farmácia e Psicologia) de uma instituição de ensino superior privada da cidade de Curitiba (PR), que responderam ao UWES-S e a um instrumento que continha dados de perfil sociodemográfico, tais como sexo, idade, estado civil, tempo de união, curso, período, turno de estudo, se tinham filhos ou não, com quem residiam, atividade laboral, tempo de dedicação aos estudos fora da faculdade, prática de atividade de lazer e física, que compuseram as variáveis articuladas ao escore de engajamento.

A escala UWES-S foi traduzida pelo Grupo de Pesquisas sobre Estresse e Burnout (Gepeb) ${ }^{22}$. O banco de dados internacional do UWES conta com tradução da escala em países como Inglaterra, Alemanha, França, Noruega, Suécia, Finlândia, Espanha, Grécia, Rússia, Portugal e Brasil, além da versão original holandesa. A versão para estudantes (UWES-S) foi traduzida para o espanhol, russo e português.

A participação dos estudantes foi voluntária, sendo convidados mediante apresentação dos objetivos e do Termo de Consentimento Livre e Esclarecido (TCLE), destinado a partici- 
pantes com 18 anos ou mais. Para a coleta de dados, a pesquisadora organizou os instrumentos de coleta de dados - UWES-S e perfil sociodemográfico - em um envelope. Neste, havia uma etiqueta com a orientação "Caro(a) estudante! Caso tenha idade menor que 18 anos, favor não responder à pesquisa. Pedimos que não se identifique, aguarde e somente entregue seu envelope após outro colega ter entregue ao monitor pesquisador. Obrigada!". Esta estratégia foi adotada para que estudantes menores de 18 anos não tivessem suas identidades expostas.

Respeitando os princípios da Resolução 466/12 do Conselho Nacional de Saúde ${ }^{23}$, os participantes foram identificados por meio de código relacionado ao curso de origem - por exemplo, "ENF" para estudantes do curso de graduação em Enfermagem, e assim por diante. O TCLE foi entregue em envelope separado. Assim, no momento das orientações iniciais, o estudante recebia dois envelopes, um com o TCLE e outro com os respectivos instrumentos. Antes de iniciar a pesquisa, foi esclarecido que a participação não acarretaria qualquer prejuízo às atividades no curso em que o estudante estava matriculado.

Antes desta etapa da pesquisa, com o intuito de padronização e também considerando o bloqueio ético na aplicação dos instrumentos por ser a pesquisadora docente da instituição de ensino, foram treinados monitores de pesquisa, totalizando 14 estudantes, que não responderam à pesquisa. A coleta de dados seguiu o agendamento organizado em planilha que continha datas, horários, períodos, número de estudantes previstos, disciplinas, docentes responsáveis. Foi realizado contato pessoal com cada docente responsável pela turma em que seria aplicada a escala e enviado, por via eletrônica, um comunicado para organização destes em suas atividades. A efetivação da coleta de dados deu-se entre os dias 14 e 24 de outubro de 2015.

Com a aplicação do UWES-S e pela análise estatística, foram propostos cinco novos modelos para este instrumento, com base no modelo original. Assim, dos cinco modelos, dois decorrem da Análise Fatorial Confirmatória, apresentando poucas sugestões de alteração na estrutura original da escala; três modelos provêm da Análise Fatorial Exploratória, que desconsiderou a existência de um modelo original e analisou o instrumento a partir da propositura de validação a um público-alvo novo, estudantes do ensino superior nas Ciências da Saúde.

Para a efetivação desta pesquisa, optou-se pelo modelo 3, cujo desenvolvimento se deu a partir do modelo 1 (original), utilizando-se o indicador de modificação, o qual aponta a(s) questão(s) que causa maior impacto ao modelo estrutural ao ser retirada ou trocada de dimensão. Ao se fazer a modificação seja troca de dimensão ou retirada - de um item, o processo se repetiu até a obtenção de um modelo considerado satisfatório, ou seja, aquele em que os indicadores RMSEA <0,07 e CFI > 0,95 com os conceitos das questões correspondessem às dimensões.

A escolha desse modelo foi baseada nos resultados obtidos pela análise estatística e conceitual. Embora outros modelos fossem mais apropriados estatisticamente, as alterações na estrutura do instrumento original seriam tão significativas que comprometeriam conceitualmente o constructo. No modelo escolhido, foram mantidas as três dimensões existentes no modelo original ${ }^{24}$, e houve alteração na quantidade de questões relacionadas a cada uma destas dimensões, passando-se de 17 para 12 questões.

Para validação, utilizou-se a Análise Fatorial Confirmatória, e foram adotados indicadores de qualidade de ajuste do modelo, como Qui-Quadrado, Raiz Quadrada Média do Erro de Aproximação (RMSEA), Índice de Ajuste Comparativo (CFI) e Goodness-of-fit (GFI). Para a comparação entre variáveis de três ou mais categorias com o escore geral de engajamento, usou-se o teste de Anova, seguido do teste post-hoc para Anova, e o teste de Tukey; já para a comparação de variáveis de duas categorias, optou-se pelo teste de Mann-Whitney.

O projeto foi aprovado em 10 de julho de 2015 sob o número 1.155.201, pelo Comitê de Ética em Pesquisa Institucional.

\section{RESULTADOS}

As características dos 537 participantes do estudo indicam 175 (32,6\%) estudantes do curso de Psicologia, 196 (36,5\%) do turno vespertino, $352(65,5 \%)$ matriculados entre o primeiro e o quarto períodos do curso, 183 (34\%) residiam com os pais. A faixa etária ficou entre 18 e 64 anos, com média de 23,16 anos de idade. Quanto ao gênero e estado civil, 426 (79,3\%) eram do sexo feminino e 455 (84,7\%) eram solteiros. Dos 78 (14,5\%) estudantes que afirmaram ser casados ou estar em união estável, 18 $(17,8 \%)$ encontravam-se nesse relacionamento havia 16 anos ou mais. Entre os participantes, $480(90,7 \%)$ responderam não ter filhos, 237 (44\%) desempenhavam alguma atividade laboral, e, destes, 132 (55,7\%) escolheram áreas correlatas aos seus cursos.

Para o tempo de dedicação aos estudos fora da faculdade, $281(53,7 \%)$ afirmaram se dedicar de uma a duas horas por dia, $83(15,9 \%)$ cerca de cinco horas ou mais por dia, e $25(4,8 \%)$ não se dedicam aos estudos fora da faculdade. Quanto ao local de residência, 420 (78,4\%) moravam em Curitiba. Para a atividade de lazer, 451 (84,5\%) expressaram que a realizam, ao contrário do resultado obtido para a prática de atividade física, em que 292 (54,6\%) estudantes afirmaram não praticar. A comparação dos escores com as variáveis levantadas na pesquisa tem os resultados apresentados nas Tabelas 1 e 2. Os estudantes de Medicina, seguidos pelos de Enfermagem, foram mais engajados. Estudantes do turno da manhã ou integral mostraram-se mais engajados.

18 REVISTA BRASILEIRA DE EDUCAÇÃO MÉDICA

$18{ }_{42(2): 14-25 ; 2018}$ 


\begin{tabular}{|c|c|c|c|c|}
\hline \multicolumn{5}{|c|}{$\begin{array}{c}\text { TABELA } 1 \\
\text { Distribuição da amostra de acordo com o curso, turno do curso e tempo de dedicação aos } \\
\text { estudos fora da faculdade e dimensões UWES-S. Curitiba (PR), 2015-2016 }\end{array}$} \\
\hline & $\begin{array}{l}\text { D1 } \\
\text { Vigor } \\
\text { média (DP) }\end{array}$ & $\begin{array}{l}\text { D2 } \\
\text { Dedicação } \\
\text { média (DP) }\end{array}$ & $\begin{array}{c}\text { D3 } \\
\text { Absorção } \\
\text { média (DP) }\end{array}$ & Geral \\
\hline \multicolumn{5}{|l|}{ Curso* } \\
\hline Biomedicina & $4,48(1,21)$ & $5,2(1,12)$ & $4,67(1,12)$ & $4,79(1,04)$ \\
\hline Enfermagem & $4,96(1,22)$ & $5,57(1)$ & $4,96(0,85)$ & $5,19(0,8)$ \\
\hline Farmácia & $4,33(1,35)$ & $5,32(1,1)$ & $4,61(1,16)$ & $4,79(1,03)$ \\
\hline Medicina & $4,78(1,19)$ & $5,29(0,97)$ & $5,1(1,01)$ & $5,12(0,89)$ \\
\hline Psicologia & $4,76(1,19)$ & $5,44(1,03)$ & $4,71(1,05)$ & $4,92(0,91)$ \\
\hline Valor de $\mathrm{p}$ & 0,017 & 0,106 & 0,005 & 0,013 \\
\hline \multicolumn{5}{|l|}{ Turno* } \\
\hline Manhã & $4,88(1,17)$ & $5,49(1,04)$ & $4,91(0,84)$ & $5,12(0,82)$ \\
\hline Integral & $4,78(1,19)$ & $5,29(0,98)$ & $5,1(1,02)$ & $5,12(0,89)$ \\
\hline Tarde & $4,47(1,25)$ & $5,23(1,12)$ & $4,67(1,12)$ & $4,8(1,04)$ \\
\hline Noite & $4,77(1,19)$ & $5,46(1,02)$ & $4,72(1,06)$ & $4,93(0,91)$ \\
\hline Valor de $\mathrm{p}$ & 0,032 & 0,131 & 0,004 & 0,019 \\
\hline \multicolumn{5}{|l|}{$\begin{array}{l}\text { Tempo de dedicação aos } \\
\text { estudos fora da faculdade* }\end{array}$} \\
\hline 1 a 2 horas/dia & $4,65(1,19)$ & $5,27(1,06)$ & $4,65(1,04)$ & $4,83(0,94)$ \\
\hline 3 a 4 horas/dia & $4,82(1,13)$ & $5,48(0,98)$ & $4,93(0,94)$ & $5,08(0,83)$ \\
\hline 5 horas ou mais & $4,78(1,24)$ & $5,55(0,88)$ & $5,35(0,98)$ & $5,35(0,87)$ \\
\hline $\begin{array}{l}\text { Não dedico horas de } \\
\text { estudo fora da faculdade }\end{array}$ & $3,5(1,41)$ & $4,64(1,53)$ & $3,78(1,1)$ & $3,94(1,08)$ \\
\hline Valor de $\mathrm{p}$ & $<0,001$ & $<0,001$ & $<0,001$ & $<0,001$ \\
\hline
\end{tabular}

Fonte: Dados da pesquisa (2016).

* Teste de Anova.

\section{TABELA 2}

Distribuição da média ( \pm desvio padrão) e intervalo de confiança de $95 \%$ para as dimensões da Utrecht Work Engagement Scale (UWES-S). Curitiba (PR), 2015-2016

\begin{tabular}{|c|c|c|}
\hline & Média (DP) & Valor geral de $p$ \\
\hline Estado civil & & 0,02 \\
\hline Casado, união estável & $5,16(0,97)$ & \\
\hline Separado, solteiro & $4,9(0,94)$ & \\
\hline Se possui filhos & & 0,042 \\
\hline Sim & $5,21(0,98)$ & \\
\hline Não & $4,91(0,95)$ & \\
\hline Prática de lazer & & 0,036 \\
\hline Sim & $4,98(0,92)$ & \\
\hline Não & $4,71(1,1)$ & \\
\hline
\end{tabular}

Fonte: Dados da pesquisa (2016).

\# Valor p correspondente ao resultado do teste de Mann-Whitney.

Para a variável período de curso, foram realizados dois tipos de análise: (1) entre estudantes de Biomedicina, Enfermagem, Farmácia e Psicologia que cursavam até o quarto período e aqueles que se encontravam a partir do quinto período, não sendo observadas diferenças significativas para o escore de engajamento entre calouros e formandos; (2) entre estudantes de Medicina do curso em fase de implantação até o quarto período, não tendo havido diferença significativa para o escore de engajamento.

Esta pesquisa concluiu que estudantes casados ou em união estável se mostraram mais engajados em suas atividades acadêmicas do que aqueles que afirmaram ser solteiros. Estudantes em relacionamento afetivo por um período de 16 anos ou mais têm maior escore de engajamento. Estudantes que têm filhos são mais engajados.

Estudantes que apresentam maior escore de engajamento com os estudos são aqueles que praticam alguma forma de atividade de lazer. Neste estudo, 451 (84,5\%) estudantes dedicam algum tempo livre a atividades de lazer, e 83 (15,5\%) não o fazem.

Foi constatado não haver diferença significativa no escore de engajamento entre estudantes que praticam ou não atividade física. Nesta pesquisa, dos 537 estudantes, 243 (45,4\%) afirmaram praticar alguma atividade física, e 292 (54,6\%) não têm o hábito da prática esportiva. 
Com base nos resultados provenientes da Análise Fatorial Confirmatória, tem-se que o instrumento UWES-S possui validade confirmatória ao ser aplicado a estudantes do ensino superior das Ciências da Saúde. Os índices de ajuste de modelo foram considerados adequados, sendo RMSEA de 0,068, tendo como parâmetro aceitável $<0,1$; para o GFI, tem-se o valor de 0,951 , sendo a referência valores entre 0 e 1, aceitável 0,9 ; e para o CFI, tendo como base o valor $\geq 0,9$, o valor obtido foi de 0,951 .

Tais valores podem ser comparados aos resultados encontrados em estudo desenvolvido na Espanha, Portugal e Holan$\mathrm{da}^{25}$ para confirmação de inúmeras hipóteses que relacionam as escalas Maslach Burnout Inventory Student Survey (MBI-SS) e Utrecht Work Engagement Scale for Students (UWES-S). Uma das hipóteses do estudo era verificar a validade da escala UWES-S com estudantes universitários. Os valores encontrados para os índices RMSEA e CFI foram, respectivamente, 0,05 e 0,97 na versão espanhola; 0,06 e 0,95 para a versão de Portugal; e 0,07 e 0,93 para a versão holandesa. Ressalte-se que este último constitui o país de origem da escala. Ao se comparar os valores obtidos para estes indicadores, entre o modelo 1 (original) e o modelo 3, nota-se que este se adapta mais ao público-alvo desta pesquisa, pois seus resultados se mostraram mais satisfatórios do que os obtidos com o modelo 1 - original (Tabela 3).

\begin{tabular}{|c|c|c|c|}
\hline \multicolumn{4}{|c|}{$\begin{array}{c}\text { TABELA } 3 \\
\text { Indicadores de qualidade de ajuste dos modelos } \\
\text { de equações estruturais (comparação entre modelo } \\
1 \text { e modelo 3). Curitiba (PR), 2015-2016 }\end{array}$} \\
\hline $\begin{array}{l}\text { Indicador de } \\
\text { qualidade }\end{array}$ & $\begin{array}{c}\text { Ponto considerado } \\
\text { adequado }\end{array}$ & $\begin{array}{l}\text { Indicadores } \\
\text { do modelo } 1\end{array}$ & $\begin{array}{l}\text { Indicadores } \\
\text { do modelo } 3\end{array}$ \\
\hline RMSEA & $<0,1$ & 0,07 & 0,068 \\
\hline CFI & $>0,9$ & 0,93 & 0,951 \\
\hline GFI & $>0,9$ & - & 0,951 \\
\hline
\end{tabular}

Fonte: Dados da pesquisa (2016) e Schaufeli et al..$^{25}$.

Comparando-se os resultados dos indicadores de qualidade obtidos na pesquisa mencionada com os deste estudo, confirma-se a validade do instrumento com estudantes universitários.

Os Diagramas 1 e 2 representam a relação estrutural entre as questões e dimensões do questionário, considerando as propostas do modelo 1 (original) e 3, sendo que para cada relação considerada estão apresentadas as estimativas padronizadas dos efeitos de cada questão. Com base nos dados estatísticos obtidos com as estimativas padronizadas é possível retirar questões ou trocá-las de dimensão.

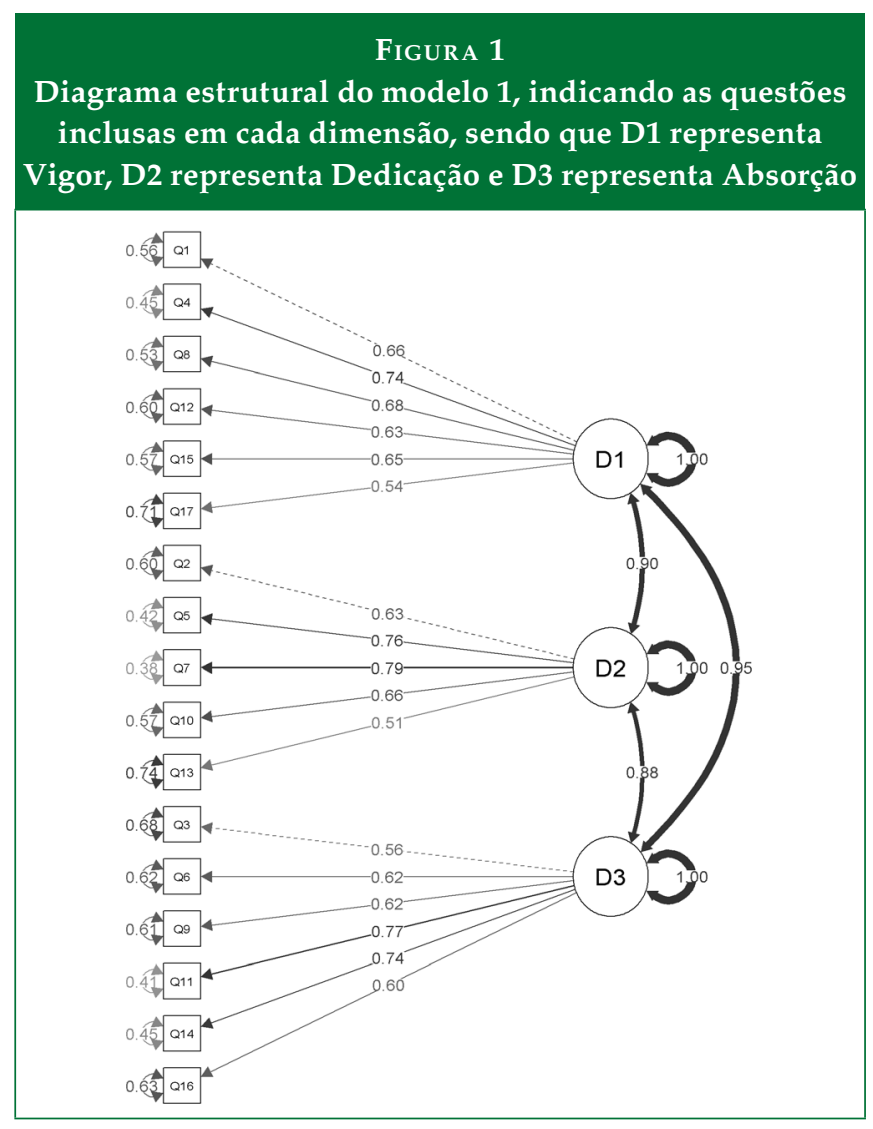

Fonte: Schaufeli et al..$^{25}$.

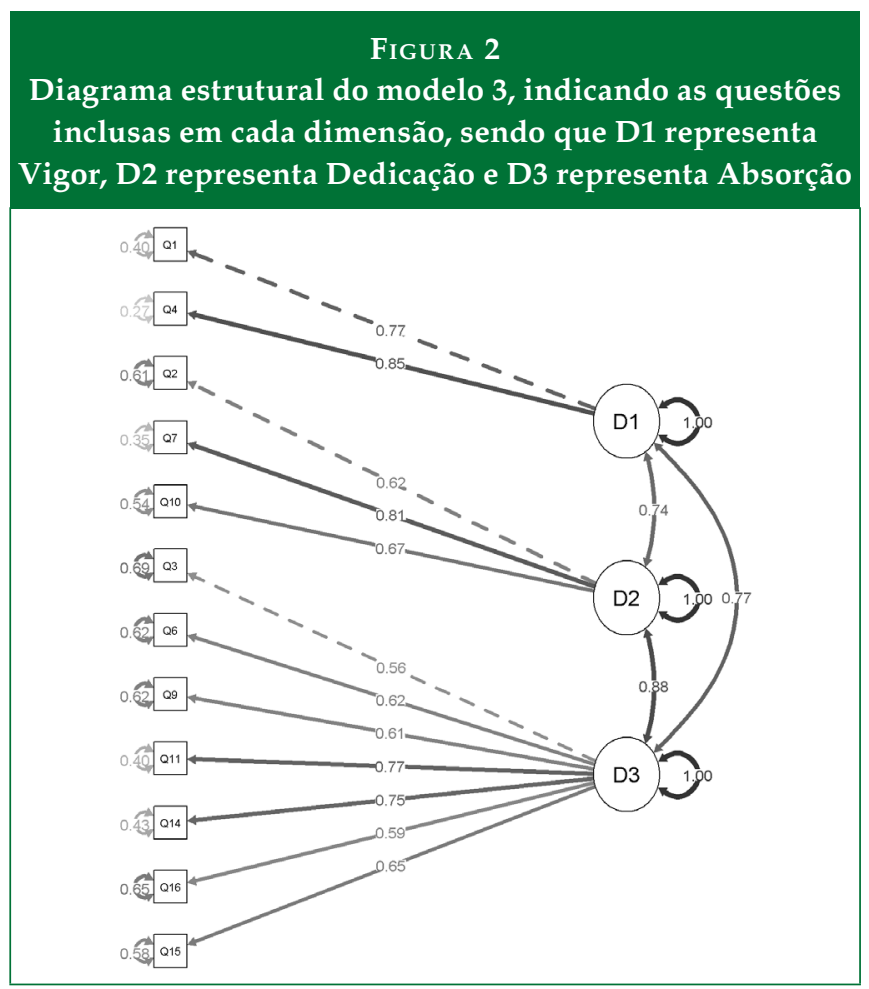

Fonte: Modelo de Silva ${ }^{21}$. 


\section{DISCUSSÃO}

O engajamento entre estudantes do ensino superior é um constructo recente, em especial relacionado à realidade brasileira. As variáveis direcionadas ao engajamento deste estudo foram idade, sexo, tempo de dedicação aos estudos, período do curso, articulação da vida acadêmica às atividades laborais, estado civil, filhos e práticas de lazer. Um estudo recente mostrou que tais variáveis podem interferir positiva ou negativamente no desenvolvimento deste constructo, em particular com estudantes de ensino superior ${ }^{16}$.

Ao se avaliarem os escores das dimensões Vigor, Dedicação e Absorção do constructo engajamento, os resultados mostraram que estudantes de Medicina e Enfermagem apresentam maior escore de Vigor e Dedicação em comparação aos demais; estudantes de Medicina apresentam maior Absorção. Com relação ao turno de estudo, obteve-se que estudantes do turno da manhã e integral exibem maior Vigor e Absorção com as atividades acadêmicas; já estudantes dos períodos iniciais de curso apresentaram maior escore de Absorção.

$\mathrm{O}$ estudo não apontou diferença significativa quanto ao gênero nas dimensões do engajamento, ao contrário de um estudo com 170 estudantes de Enfermagem de uma instituição de ensino superior de Málaga (Espanha), que mostrou que o escore de engajamento apresentado por estudantes do sexo feminino foi significativamente maior que o do masculino ${ }^{26}$.

Em relação ao tempo de dedicação aos estudos, os estudantes que dedicam mais tempo aos estudos/atividades acadêmicas fora do âmbito da faculdade (de três a cinco horas ou mais) apresentam maior escore de engajamento. $\mathrm{O}$ mesmo também foi visto em outro estudo segundo o qual estudantes que dedicam maior tempo a seus estudos, não adiam decisões, dificilmente se distraem e não cometem erros em tarefas que lhes são atribuídas apresentam alto escore de engajamento ${ }^{26}$.

Fior et al. ${ }^{27}$ destacaram que nem toda forma de envolvimento pode ser associada a resultados acadêmicos positivos, mas, sim, aquelas relacionadas à dedicação aos estudos, ao número de horas dedicadas e ao grau de interesse no curso.

Sobre o período do curso, esta pesquisa não encontrou diferença no escore de engajamento entre estudantes dos cursos de Enfermagem, Farmácia, Biomedicina e Psicologia no que tange a serem calouros ou formandos e, tampouco, na análise entre estudantes de Medicina que se encontravam no primeiro ano e aqueles do segundo ano da graduação. O curso de Medicina encontrava-se em fase de implantação, tendo, à época da coleta dos dados, estudantes até o quarto período (correspondente ao segundo ano). Jang et al. ${ }^{13}$ concluíram que os níveis de engajamento de estudantes podem se mostrar elevados ao longo dos semestres quando os docentes assumem uma postura de incentivo à autonomia e ao alcance da satisfação acadêmica.

Ao refletir acerca de estudantes das Ciências da Saúde, percebe-se que estes se deparam constantemente com inúmeros enfrentamentos, como a responsabilidade de assistir outras pessoas, o limite entre doença e morte, fragilidades emocionais, necessidade de autonomia na tomada de decisões, ritmo intenso de vida estudantil, experiências em situações de atendimento de urgência, confronto com a morte, além do pouco tempo para lazer e família ${ }^{2028,29,30}$. Depreende-se que tais situações, quando não reconhecidas e manejadas de forma adequada, podem impactar o nível de engajamento e tendem a afetar a saúde, o processo de aprendizagem, a satisfação com os estudos e, consequentemente, a qualidade da atenção dispensada aos que dependem de seu aprendizado,

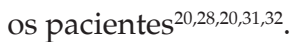

Com vistas à manutenção do engajamento pelo estudante, é importante o equilíbrio entre fatores sociais e psicológicos, uma vez que, quando engajado, o estudante de ensino superior se sente muito bem, tanto mental quanto socialmente, o que impacta diretamente a qualidade de suas atividades acadêmicas ${ }^{7}$. Os fatores vinculados a este conceito podem ser o apoio social e familiar, o desempenho nas atividades acadêmicas, auxílio de colegas e amigos, recursos pessoais e psicológicos do estudante, valores e crenças, estratégias de enfrentamento utilizadas, confiança em seus atos, recursos e demandas da instituição de ensino, resiliência ${ }^{9,28}$.

Bublitz et al..$^{33}$ discutem o apoio familiar, afirmando que estudantes que residem com seus familiares durante a formação acadêmica demonstram maior facilidade para se adaptarem às mudanças impostas pelo ingresso no ensino superior, uma vez que as famílias tendem a oferecer maior suporte social e emocional ao estudante, conferindo tranquilidade na tomada de decisões e nos momentos de estresse. Corroborando esta visão, Dias e Sá ${ }^{34}$ e Mesa ${ }^{35}$ apontaram que a família tem papel fundamental na vida do estudante, por constituir uma área de significação relevante ao indivíduo, além de funcionar como um sistema de vigilância para possíveis situações de estresse. Trazem ainda que a família compartilha com o estudante os êxitos e os fracassos vividos durante o ensino superior, considerando que esta tem significado importante para qualquer pessoa e funciona como um dos pilares do indivíduo, culminando na melhora do desempenho acadêmico do estudante ${ }^{35,36}$.

Um fator importante a ser considerado na atualidade é a duplicidade de papéis desempenhada por estudantes de ensino superior quando associam trabalho e estudo, exigindo do docente e da instituição de ensino um olhar atento sobre seu 
desenvolvimento. Tal decisão tem impacto direto no resultado final de suas atividades acadêmicas, pois demanda maior energia e esforço, ocupando horas que seriam dedicadas à família, ao lazer e à atividade física ${ }^{37}$.

Em relação à variável trabalho, o estudo revelou não existir diferença no nível de engajamento entre estudantes que trabalham e aqueles que não trabalham. Todavia, Caballero et $a l .{ }^{38}$ notaram que estudantes que trabalham tendem a se envolver mais com a demanda acadêmica, demonstrando maior dedicação, possivelmente pelo esforço de conseguir conciliar trabalho e estudo, o que pode resultar em maior entusiasmo, inspiração, orgulho e desafio pessoal para com as atividades acadêmicas. Obter sucesso tanto no trabalho quanto na vida acadêmica pode servir como motivador para que esse estudante trabalhador seja cada vez mais engajado ao longo de sua formação.

Quanto ao estado civil, percebeu-se neste estudo que os estudantes que afirmaram ser casados ou estar em união estável mostraram-se mais engajados com suas atividades acadêmicas do que aqueles que afirmaram não estar em um relacionamento afetivo no momento da pesquisa. Gárcia Rodríguez et al. ${ }^{26}$ observaram em pesquisa desenvolvida com 170 estudantes de Enfermagem, que a dimensão 3 (Absorção) foi mais significativa para os estudantes que são casados ou se encontram em uma união estável em comparação aos solteiros.

Com relação a ter ou não filhos, os estudantes deste estudo que declararam ter filhos são mais engajados com as atividades acadêmicas em comparação àqueles que não têm filhos.

Este estudo constatou que estudantes que afirmaram praticar alguma forma de lazer apresentam escore maior de engajamento do que aqueles que responderam não dedicar parte do tempo a alguma prática de lazer. Em estudo com 134 estudantes de uma universidade do interior de São Paulo, percebeu-se que a grande maioria dedicava, em média, uma hora a duas horas e meia a atividades de lazer, categorizadas por áreas de interesse, como artística, intelectual, social, virtual e viagem. Mesmo com tais resultados, a pesquisa apontou um perfil de estudantes com pouco tempo para atividades de lazer, sendo a falta de tempo a maior barreira verificada ${ }^{39}$. Assim, é premente uma reflexão acerca do tempo dedicado ao lazer por estudantes universitários, pois a maioria trabalha durante o dia e estuda à noite ${ }^{40,41}$.

Quando observados os resultados deste estudo para a variável prática de atividade física, percebe-se que não há diferença significativa no escore de engajamento entre estudantes que praticam atividade física comparados aos que declararam não praticar. Considerando que a vida acadêmica demanda altos níveis de energia, julga-se a prática regular de atividade física uma estratégia importante na prevenção de inúmeros agravos à saúde e de promoção da saúde do estudante ${ }^{30,42,43}$. Pesquisas desenvolvidas com estudantes universitários revelam que mais de $50 \%$ são sedentários ${ }^{42,44}$ e, em algumas destas, sem distinção quanto ao sexo. Os pesquisadores identificaram que um dos fatores que pode contribuir para este comportamento, em especial advindo de estudantes da área da saúde, é o exíguo tempo livre entre as demandas acadêmicas, servindo de fator predisponente ao estresse, a doenças cardiovasculares e, relacionando ao tema deste estudo, à redução no nível de engajamento com atividades acadêmicas ${ }^{45,46,43}$.

Todavia, outros estudos mostram que a prática regular de atividades físicas contribui para a melhora das funções cognitivas - percepção, aprendizagem, memória, atenção, vigilância, raciocínio e capacidade de solucionar problemas -, como também do desempenho acadêmico, além dos benefícios físi$\cos$, emocionais e nas interações sociais ${ }^{47,48,49}$. As hipóteses para tais efeitos estão relacionadas a aumento do fluxo sanguíneo regional, a maior atividade de neurotransmissores sinápticos, a promoção de adaptações em estruturas cerebrais e plasticidade sináptica, além de atividade antioxidante ${ }^{45,47,48}$.

O uso deste constructo permite determinar o nível de engajamento dos estudantes com as atividades acadêmicas, considerando que é uma das variáveis essenciais na predição do rendimento acadêmico. Esta avaliação pode ser efetuada em momentos pontuais ou ao longo da formação.

Ao mesmo tempo, esse novo construto é de potencial importância para medir a quantidade e a qualidade de energia despendida na efetivação das tarefas acadêmicas para o aperfeiçoamento educacional, organizacional e pessoal, e para o estabelecimento de estratégias eficazes para a manutenção do engajamento do estudante, visando à qualidade de sua formação. Desta forma, conhecer este constructo aplicado aos estudantes de ensino superior da área de saúde possibilita um novo olhar sobre o contexto acadêmico, oferece visibilidade ao estudante, coloca-o no centro do processo de ensino e aprendizagem e considera suas condutas positivas. O docente assume o papel de mediador e, juntamente com a IES, é o sustentáculo deste processo no âmbito da saúde, o que vai ao encontro das Diretrizes Curriculares do Curso de Graduação de Medicina de 2014.

As instituições de ensino desempenham um papel essencial no desenvolvimento acadêmico e, consequentemente, no nível de engajamento do estudante por intermédio da proposição de projetos pedagógicos e matrizes curriculares que propiciem o comprometimento dos estudantes, do oferecimento de estruturas de suporte e da disponibilidade de recursos humanos, como docentes e outros colaboradores aptos a viabili- 
zarem experiências com as quais os estudantes possam se envolver e desenvolver. Ao implementarem medidas de apoio, o curso e a IES auxiliam na adaptação do estudante no ensino superior para que este possa lidar com as pressões e desafios acadêmicos, sociais e emocionais, e obter um desenvolvimento harmonioso e global.

Utilizar instrumentos para mensurar simultaneamente o estresse e o desempenho acadêmico de estudantes das Ciências da Saúde, fornecendo um panorama amplo dos aspectos positivos e negativos envolvidos no processo de adaptação do estudante ao universo acadêmico, pode contribuir para a detecção precoce de problemas, principalmente relacionados à sua saúde mental.

\section{CONCLUSÃO}

Os estudantes que participaram desta pesquisa mostraram-se engajados, sobretudo aqueles que cursam Medicina e Enfermagem, casados, com filhos, que dedicam grande parte de seu tempo aos estudos, que trabalham e que praticam atividades de lazer. Não foram encontradas diferenças significativas no escore geral e nas dimensões do UWES-S para as variáveis sexo, período/ano do curso e atividade física.

Pode-se concluir que o Questionário do Bem-Estar e Trabalho para Estudantes (UWES-S) mostrou-se um instrumento confiável e válido para medir o escore de engajamento de estudantes do ensino superior nas Ciências da Saúde. Como ainda não foram desenvolvidos estudos que relacionem este conceito à atividade acadêmica de estudantes da área da saúde, esta pesquisa é a primeira no Brasil a abordar esta interação, instigando novas pesquisas que utilizem o engajamento, além de contribuir para o desenvolvimento científico.

Assim, depreende-se que o tema engajamento entre estudantes do ensino superior nas Ciências da Saúde necessita de aprofundamento por meio de outras pesquisas que possibilitem a construção e validação de concepções teóricas com o propósito de estabelecer o estado da arte sobre o assunto em constante evolução e repensar estratégias para manutenção e/ou incentivo ao engajamento dos estudantes na vida universitária.

\section{PERSPECTIVAS FUTURAS}

O preparo para perceber o engajamento ou não do estudante e avaliar o escore de engajamento no decorrer da formação como aspecto rotineiro requer investimento no contínuo desenvolvimento docente para atualizar e manter o potencial das metodologias ativas como motivador para os estudos, bem como o estabelecimento de práticas de maior envolvimento nos estudos fora da sala de aula, estratégias relacionais motivadoras e apoio institucional. Deve-se ampliar o número de participantes em novas pesquisas a fim de possibilitar a generalização dos resultados, bem como incluir estudantes de instituição de ensino superior pública, considerando o contexto da instituição e as variáveis relacionadas aos estudantes.

\section{LIMITAÇÕES DO ESTUDO}

O estudo foi realizado em uma única instituição privada de ensino da área de saúde, havendo a necessidade de novos estudos com distintas universidades públicas e privadas. A escala UWES-S avalia o engajamento dos estudantes com as três dimensões propostas, podendo-se ampliar ou complementar as investigações com o uso de outras escalas de mensuração de rendimento escolar ou qualidade de vida.

\section{REFERÊNCIAS}

1. Caballero, C, Llanos, RA, Sañudo, JP. Burnout, engagement y rendimiento académico entre estudiantes universitarios que trabajan y aquellos que no trabajan. Revista Psicogente. 2006; 9(16):11-27.

2. Cunha, SM, Carrilho, DM. O processo de adaptação ao ensino superior e o rendimento acadêmico. Psicologia Escolar e Educacional. 2005; 9(2):215-24.

3. Bento, A, Mendes, G. A transição do ensino secundário para o ensino superior: factores contributivos para uma boa adaptação e relação com o sucesso académico universitário [Internet]. In: Anais do IX Congresso da Sociedade Portuguesa de Ciências da Educação: 2007; Porto, Portugal. Porto: Legis Editora; 2007. p.245-51. [acesso em 2015 Mar 29]. Disponível em: http://digituma.uma.pt/ bitstream/10400.13/50/1/BentoAdaptação\%20dos\%20 alunos\%20à\%20Universidade.pdf

4. Cabral, J, Matos, PM. Preditores da adaptação à universidade: o papel da vinculação, desenvolvimento psicossocial e coping. Psychologica. 2010; 52(1):55-77.

5. Teixeira, MAP, Dias, ACG, Wottrich, SH, Oliveira, AM. Adaptação à universidade em jovens calouros. Revista Semestral da Associação Brasileira de Psicologia Escolar e Educacional. 2008; 12(1):185-202.

6. Porto-Martins, PC, Basso-Machado, PG, Benevides-Pereira, AMT. Engagement no trabalho: uma discussão teórica. Fractal: Revista de Psicologia. 2013; 25(3):629-44.

7. Schaufeli, W, Bakker, A. UWES: Work Engagement Scale Preliminary Manual. Utrecht Holanda: Utrecht University, version 1, November, 2003.

8. Bakker, AB, Demerouti, E, Sanz-Vergel, AI. Burnout and work engagement: the JD-R approach. Annual Rewiew of Organization Psychology and Organizational Behavior. 2014; 1:389-11. 
9. Machado, PGB, Porto-Martins, PC, Amorin, C. Engagement no trabalho entre profissionais da educação. Revista Intersaberes. 2012; 7(13):193-14.

10. Scorsolini-Comin, F, Santos, MA. Psicologia positiva e os instrumentos de avaliação no contexto brasileiro. Psicologia: Reflexão e Crítica. 2010; 23(3):440-48.

11. González-Romá, V, Schaufeli, W, Bakker, AB, Lloret, S. Burnout and engagement: independent factors or opposite poles? Journal of Vocational Behavior. 2006; 68:165-74.

12. Blázquez, BO, Sarto, SB, Hoyo, YL. Estrés y otros factores psicológicos associados em estudiantes de fisioterapia. Fisioterapia. 2011; 33(1):19-24.

13. Jang, H, Kim, EJ, Reeve, J. Why students become more engaged or more disengaged during the semester: a self-determination theory dual-process model. Learning and Instruction. 2016; 43:27-38.

14. Llorens, S, Schaufeli, W, Bakker, AB, Salanova, M. Does a positive gain spiral of resources, efficacy beliefs and engagement exist? Computers in human behavior. 2004; 23:825-41.

15. Wang, M, Fredricks, J. The reciprocal links between school engagement, youth problem behaviors, and school dropout during adolescence. Child Development. 2014; 85(2):722-37.

16. Fredricks, JA, Filsecker, M, Lawson, MA. Student engagement, context, and adjustment: Addressing definitional, measurement, and methodological issues. Learning and Instruction. 2016; 43: 1-4.

17. Soares, APC, Vasconcelos, RM, Almeida, LS. Adaptação e satisfação na universidade: apresentação e validação do questionário de satisfação acadêmica. In: Pouzada, AS, Almeida, LS, Vasconcelos RM, orgs. Contextos e dinâmica da vida acadêmica. Braga, Portugal: Universidade do Minho; 2002. p.153-65.

18. Contreras, MP, Villalobos, CEP. Relación del engagement académico con características académicas y socioafectivas en estudiantes de odontologia. Educación Médica Superior. 2014; 28(2):199-15.

19. Goldberg, DP; Williams, P. Cuestionario de salud general GHQ (General Health Questionnaire). Barcelona: Elsevier-Masson. 1996.

20. Liébana-Presa, C, Fernández-Martínez, ME, Gándara, AR, Muñoz-Villanueva, MC, Vázquez-Casares, AM, Rodríguez-Borrego, MA. Psychological distress in health sciences college students and its relationship with academic engajamento. Revista da Escola de Enfermagem da USP. 2014; 48(4):715-22.

21. Silva JOM. Engagement entre estudantes do ensino superior das ciências da saúde. Curitiba; 2016. Mestrado [Dissertação] - Faculdades Pequeno Príncipe.
22. Angst, R, Benevides-Pereira, AMT, Porto-Martins, PC. UWES - Utrecht Work Engagement Scale (Escala de Engajamento no Trabalho de Utrecht) [Internet]. Grupo de Estudos e Pesquisa sobre Estresse e Burnout (GEPEB): Pontifícia Universidade Católica; 2009.

23. Resolução $n^{\circ} 466$, de 12 de dezembro de 2012. Dispõe sobre a pesquisa e testes em seres humanos. Diário Oficial da União, 13 Jun 2013.

24. Schaufeli, W, Bakker, UWES - Utrecht Work Engagement Scale (Escala de Engajamento no Trabalho de Utrecht) [Internet]. Utrecht: Utrecht University; 2008 [acesso em 14 Mar 2015]. Disponível em: http://www.wilmarschaufeli. nl/downloads/test-manuals-2/

25. Schaufeli, WB, Martínez, IM, Pinto, AM, Salanova, M, Bakker, AB. Burnout and engagement in university students: a cross-national study. Journal of Cross-Cultural Psychology. 2002; 33(5):464-81.

26. García Rodríguez, JJ, Labajos Manzanares, MT, Fernández Luque, F. Los estudiantes de grado em enfermeria y su compromisso con los estudios. Enfermeria Global. 2015; 38:169-77.

27. Fior, CA; Mercuri, E.; Silva, D. Evidências de validade da escala de envolvimento acadêmico para universitários. Avaliação Psicológica. 2013; 12(1):81-89.

28. Binotto, M; Schaurich, D. Estresse em acadêmicos do curso de enfermagem: uma abordagem qualitativa. REUOL - Revista de Enfermagem (UFPE online). 2010; 4(3):1371-1376.

29. Schaufeli, W, Martínez, IM, Pinto, AM, Salanova, M, Bakker, $\mathrm{AB}$. Burnout and engagement in university students. Journal off Cross-Cultural Psychology. 2002; 33(5):464-81.

30. Bublitz, S; Freitas, EO; Kirchhof, RS; Lopes, LFD; Guido, LA. Estressores entre acadêmicos de enfermagem de uma universidade pública. Revista de Enfermagem UERJ (Rio de Janeiro). 2012; 20(esp.2):739-745.

31. Tomaschewski-Barlem, JG, Lunardi, VL, Lunardi, GL, Barlem, ELD, Silveira, RS, Vidal, DAS. Síndrome de burnout entre estudantes de graduação em enfermagem de uma universidade pública. Revista Latino- Americana de Enfermagem. 2014; 22(6):934-41.

32. Costa, EFO, Santos, SA, Santos, ATRA, Melo, EV, Andrade, TM. Burnout syndrome and associated factors among medical students: a cross-sectional study. Clinics. 2012; 67(6):573-79.

33. Bublitz, S, Guido, LA, Kirchhof, RS, Neves, ET, Lopes, LFD. Perfil sociodemográfico e acadêmico de discentes de enfermagem de quatro instituições brasileiras. Revista Gaúcha de Enfermagem. 2015; 36(1):77-83.

34. Dias, D; Sá, MJ. O estatuto sociocultural familiar como vetor da decisão vocacional: promessas e (des)ilusões da 
entrada na educação superior. Revista Brasileira de Orientação Profissional. 2014; 15(1):51-64.

35. Mesa, IAS. La família y su participación em la universalización de la Educación Superior. Revista Cubana de Educación Médica Superior. 2013; 27(1):54-63.

36. Daltro, MR; Pondé, MP. Atenção psicopedagógica no ensino superior: uma experiência inovadora na graduação em medicina. Construção Psicopedagógica (São Paulo). 2011; 19(18):104-123.

37. Silva, VLS; Chiquito, NC; Andrade, RAPO; Brito, MFP; Camelo, $\mathrm{SHH}$. Fatores de estresse no último ano do curso de graduação em enfermagem: percepção dos estudantes. Revista de Enfermagem UERJ (Rio de Janeiro). 2011; 19(1):121-126.

38. Caballero, CC, Hederich, C, García, A. Relación entre burnout y engajamento académicos com variables sociodemográficas y académicas. Psicología Desde El Caribe. 2015; 32(2):254-67.

39. Buzacarini, C, Corrêa, EA. Lazer dos estudantes universitários. Conexões: revista da Faculdade de Educação Física da UNICAMP. 2015; 13(2):15-28.

40. Santos, LR, Ribeiro, JP, Guimarães, L. Estudo de uma escala de crenças e de estratégias de coping através do lazer. Análise Psicológica. 2003; 21(4):441-51.

41. Martoni, FR, Schwartz, GM. O lazer na vida do estudante universitário. Revista Digital Efdeportes [on line]. 2006 [capturado em 14 jul. 2016]; 97. Disponível em: http:// www.efdeportes.com/efd97/lazer.htm

42. Santos, LR, Brito, ECC, Neto, JCGL, Alves, LEP, Alves, LRA, Freitas, RWJF. Análise do Sedentarismo em estudantes universitários. Revista de Enfermagem da UERJ (Rio de Janeiro). 2014; 22(3):416-421.

43. Maciel, ES, Sonati, JG, Modeneze, DM, Vasconcelos, JS, Vilarta, R. Consumo alimentar, estado nutricional e nível de atividade física em comunidade universitária brasileira. Revista de Nutrição (Campinas). 2012; 25(6):707-718.

44. Santos, JJA, Saracini, N, Silva, WC, Guilherme, JH, Costa, TA, Silva, MRAG. Estilo de vida relacionado à saúde de estudantes universitários: comparação entre ingressantes e concluintes. ABCS Health Science. 2014; 39(1):17-23.

45. Pirajá, GA, Sousa, TF, Fonseca, SA, Barbosa, AR, Nahas, MV. Autoavaliação positiva de estresse e prática de ativi- dades físicas no lazer em estudantes universitários brasileiros. Revista Brasileira de Atividade Física e Saúde (Pelotas). 2013; 18(6): 740-749.

46. Mascena, GV, Cavalcante, MSB, Marcelino, GB, Holanda, SA, Brandt, CT. Fatores de risco cardiovasculares em estudantes da Faculdade de Ciências Médicas de Campina Grande. Medicina Ribeirão Preto. 2012; 45(3):322-28.

47. Merege, CAA, Alves, CRR, Sepúlveda, CA, Costa, AS, Junior, AHL, Gualano, B. Influência do exercício físico na cognição: uma atualização sobre mecanismos fisiológicos. Revista Brasileira de Medicina do Esporte. 2014; 20(3): 237-241.

48. Cooper, SB, Bandelow, S, Nute, ML, Dring, KJ, Stannard, RL, Morris, JG, Nevill, ME. Sprint-based exercise and cognitive function in adolescentes. Preventive Medicine Reports. 2016; 4:155-161.

49. Antunes, HKM, Santos, RF, Cassilhas, R, Santos, RVT, Bueno, OFA, Mello, MT. Exercício físico e função cognitiva: uma revisão. Revista Brasileira de medicina do esporte. 2006; 12(2): 108-114.

\section{CONTRIBUIÇÃO DOS AUTORES}

Juliana Ollé Mendes da Silva e Ivete Palmira Sanson Zagonel participaram de todas as etapas da pesquisa e da elaboração do artigo. Gerson Alves Pereira Junior e Izabel Cristina Meister Martins Coelho participaram da redação e revisão do texto. Gledson Luiz Picharski participou da análise estatística.

\section{CONFLITO DE INTERESSES}

Declaramos que nenhum dos autores deste estudo possuem qualquer tipo de interesse de natureza pessoal, comercial, política, acadêmica ou financeira ou outros que configurem Conflito de Interesses.

\section{ENDEREÇO PARA CORRESPONDÊNCIA}

Juliana Ollé Mendes da Silva

Rua Professor José Nogueira dos Santos, 1942

Boqueirão, Curitiba (PR)

CEP: 81670-160

E-mail: juollesilva@gmail.com 